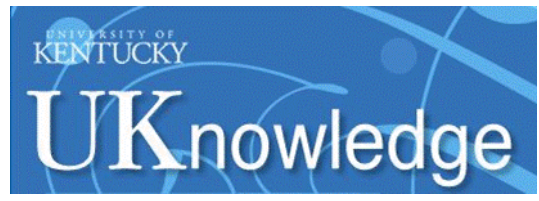

University of Kentucky

UKnowledge

\title{
The Future (or Lack of Future) of Personalized Prescription in Psychiatry
}

Jose de Leon

University of Kentucky, jdeleon@uky.edu

Follow this and additional works at: https://uknowledge.uky.edu/psychiatry_facpub

Part of the Pharmacology Commons, and the Psychiatry and Psychology Commons

Right click to open a feedback form in a new tab to let us know how this document benefits you.

\section{Repository Citation}

de Leon, Jose, "The Future (or Lack of Future) of Personalized Prescription in Psychiatry" (2009).

Psychiatry Faculty Publications. 43.

https://uknowledge.uky.edu/psychiatry_facpub/43

This Article is brought to you for free and open access by the Psychiatry at UKnowledge. It has been accepted for inclusion in Psychiatry Faculty Publications by an authorized administrator of UKnowledge. For more information, please contact UKnowledge@lsv.uky.edu. 


\section{The Future (or Lack of Future) of Personalized Prescription in Psychiatry \\ Digital Object Identifier (DOI) \\ http://dx.doi.org/10.1016/j.phrs.2008.10.002}

\section{Notes/Citation Information}

Published in Pharmacological Research, v. 59, issue 2, p. 81-89.

(c) 2008 Elsevier Ltd. All rights reserved.

This manuscript version is made available under the CC-BY-NC-ND 4.0 license

http://creativecommons.org/licenses/by-nc-nd/4.0/ 
(C) 2008 Elsevier Ltd. All rights reserved.

This manuscript version is made available under the CC-BY-NC-ND 4.0 license

http://creativecommons.org/licenses/by-nc-

nd $/ 4.0 /$ 


\section{Accepted Manuscript}

Title: The future (or lack of future) of personalized prescription in psychiatry

Author: Jose de Leon

PII: $\quad$ S1043-6618(08)00198-9

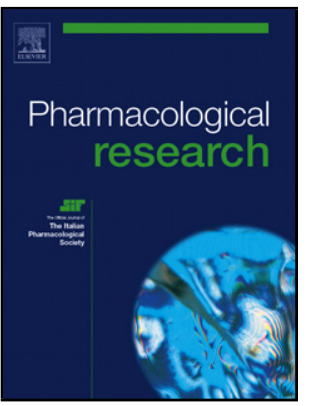

DOI: doi:10.1016/j.phrs.2008.10.002

Reference: YPHRS 1940

To appear in: Pharmacological Research

Received date:

8-10-2008

Accepted date:

9-10-2008

Please cite this article as: de Leon $\mathrm{J}$, The future (or lack of future) of personalized prescription in psychiatry, Pharmacological Research (2008), doi:10.1016/j.phrs.2008.10.002

This is a PDF file of an unedited manuscript that has been accepted for publication. As a service to our customers we are providing this early version of the manuscript. The manuscript will undergo copyediting, typesetting, and review of the resulting proof before it is published in its final form. Please note that during the production process errors may be discovered which could affect the content, and all legal disclaimers that apply to the journal pertain. 


\title{
Perspective
}

\section{The future (or lack of future) of personalized prescription in psychiatry}

\author{
Jose de Leon, M.D., ${ }^{\text {a,b }}$
}

${ }^{a}$ University of Kentucky Mental Health Research Center at Eastern State Hospital, and University of Kentucky Colleges of Medicine and Pharmacy, Lexington, Kentucky.

${ }^{\mathrm{b}}$ Psychiatry and Neurosciences Research Group (CTS-549), Institute of Neurosciences, University of Granada, Granada, Spain.

Corresponding address: Jose de Leon, M.D., Mental Health Research Center at Eastern State Hospital, 627

West Fourth St., Lexington, KY 40508. Phone (859) 246-7563. Fax (859) 246-7019. E-mail:

jdeleon@uky.edu

Disclosure/conflict of interest: Dr. de Leon is currently a co-investigator in a NIH Small Business Innovation Research Grant 2 R44 MH073291-02 “DNA Diagnostics for Minimizing Metabolic SideEffects of Antipsychotics" awarded to Genomas Inc. In the past three years (since July $1^{\text {st }}, 2005$ ), Dr. de Leon has received researcher-initiated grants from Roche Molecular Systems, Inc., and from Eli Lilly (the latter as co-investigator) and was on the advisory board of Roche Molecular Systems, Inc. (2006). He personally develops his presentations for lecturing and has never lectured using any pharmaceutical company presentations. In the last three years (since July $1^{\text {st }}, 2005$ ) his lectures have been supported seven times by Roche Molecular Systems, Inc. (once in 2005 and six times in 2006), twice by Eli Lilly (2006), once by Janssen (2006), and once by Bristol-Myers Squibb (2006). He has never been a consultant and has no other financial arrangements with pharmacogenetic or pharmaceutical companies nor owns any of their stocks.

Acknowledgments: The author thanks Lorraine Maw, M.A., for editorial assistance. 
ABSTRACT

Rapid technological advances in genetics have created conceptual chaos regarding the genetics of drug response. Terms for differing concepts are used interchangeably: pharmacogenetics with pharmacogenomics, personalized medicine with personalized prescription. Biomarker has many definitions. The author prefers the concept of personalized prescription and uses it with implications beyond pharmacogenetics by considering all scientific information valid for prescribing medication. Genetics may not be crucial for all drugs. In this comprehensive view, clinicians must consider genetic, environmental and personal variables when prescribing medication and incorporate some basic pharmacological principles: 1) safety and efficacy, 2) pharmacokinetics and pharmacodynamics, 3) therapeutic window and prescriber's role, and 4) idiosyncratic and dose-related adverse drug reactions. Personalized prescription in the clinical environment can be expressed in two main ways: as personalized selection of the drug and as personalized dosing.

The future, or lack of future, of personalized drug selection and of personalized dosing in psychiatry is reviewed. Currently, the author thinks that, in psychiatry, pharmacogenetic tests have some potential in two areas: 1) excluding some drugs for some unusual patients (HLA-B*1502 genotyping in Asians for carbamazepine), and 2) using pharmacokinetic genes for personalizing dosing in narrow therapeutic window drugs. In the short term, there is dubious potential for other pharmacogenetic tests and no potential for pharmacogenetic testing to ascertain the best drug for each patient. Personalized dosing has immediate application if one understands it as the use of our current scientific knowledge of genetic, environmental and personal variables to determine dosing; its sole requirement is well-trained psychiatrists. 


\section{Introduction}

The end of the $20^{\text {th }}$ century brought new hopes of a revolution in medicine based on our advancing knowledge of the human genome [1,2]. Personalizing pharmacological treatment has been proposed as the driving force for implementing genetic advances in primary care $[3,4]$. The first pillar of the genetic or genomic revolution was the development of new technologies that permitted parallel genetic testing (testing for many genetic variations) by using computerized genotyping [5]. Currently, more advanced forms of these types of DNA microarray technologies [6], including the Illumina BeadArray platform [7], allow testing of more than one million single nucleotide polymorphisms (SNP) at a cost of less than $\$ 1,000$ per sample, and the price is rapidly decreasing. The second pillar of this revolution was the mapping of the human genome which was completed in 2000 [8] and published in 2001 [9,10].

The first surprise stemming from the mapping of the human genome was that the human genome has only 30,000 genes (versus more than 100,000 expected) [11]. Nevertheless, 30,000 genes produce millions of genetic variations, including almost 9 million SNPs [12]. One would think that the reduction in the number of genes from the 100,000 expected to 30,000 found would simplify the task. However, it has become evident we do not yet know the function of approximately one third of human genes [13].

Recent developments have proven how naïve it was to think that the mapping of the human genome was going to change medicine in the short term. It is becoming clear that other types of genetic variations such as deletions or duplications, the so-called copy number variations (CNV), may have been neglected [14]. Unfortunately, many of the current platforms and systems used for genotyping pay attention mainly to SNPs and give little attention to CNVs. But CNVs may be important for pharmacogenomics [15]; it was a pharmacogenetic gene in which the clinical relevance of gene multiplications was discovered [16]. Less common genetic variations such as microsatellite polymorphisms and translocations, inversions, and substitutions may have some relevance in pharmacogenomics [17]. Finally, the relevance of epigenetics to pharmacogenetic response in humans is 
not well understood [18], but it is important to know that a fly's drug tolerance to an anesthetic appeared to be mainly caused by epigenetic mechanisms [19].

The next four sections attempt to lessen the conceptual chaos that the rapid technological advances in genetics have created in the area of the genetics of drug response. The concepts of pharmacogenetics/pharmacogenomics, biomarkers, personalized medicine/personalized prescription are frequently used interchangeably but are not exactly the same in the author's view.

\section{The concepts of pharmacogenetics and pharmacogenomics in the literature}

Vogel [20] first coined the term pharmacogenetics, which for many years was associated with the genetics of pharmacokinetic factors, particularly of metabolic enzymes. In the 1990s, the concept of pharmacogenomics was also introduced in the literature. In 2001, Pirmohamed reported that pharmacogenetics had been defined as the study of variability in drug response, while the term pharmacogenomics was a broader term encompassing all genes in the genome that may determine drug response. He considered the distinction as arbitrary in that both terms were frequently used interchangeably [21]. The perspective of this author is that pharmacogenetics usually is used in the context of studies attempting to verify the influence of candidate genes, while pharmacogenomics is frequently used in an exploratory context such as genome-wide scans. Roses [22] made an important distinction between two types of pharmacogenetics. Safety pharmacogenetics is aimed at avoiding adverse drug reactions (ADRs). Efficacy pharmacogenetics is meant to predict response to medications.

\section{The concept of biomarker in the literature}

The term biomarker has many definitions, including the one proposed by Wagner [23], "a characteristic that is objectively measured and evaluated as an indicator of normal biological processes, pathogenic processes, or pharmacological response(s) to a therapeutic intervention." Some, then, are pharmacogenomic biomarkers and others are not $[17,24]$. Biomarkers play an important role in the pharmaceutical industry and are assuming an ever greater role in drug discovery and development [25]. 
The concept of biomarkers is introduced here, because in the spring of 2005 the Food and Drug Administration (FDA) developed guidelines for pharmaceutical companies on the collection and inclusion of genetic information for drug applications [26]. According to the FDA [26], genetic variants of two metabolic enzymes, CYP2D6 and Thiopurine S-Methyltranseferase (TPMT), were considered to be well established and, therefore, valid biomarkers. When submitting an investigational new drug (IND) application to the FDA, pharmaceutical companies must send relevant data on "valid biomarkers", although other pharmacogenetic data can be submitted voluntarily [27].

Since the "genomic" boom, technological advances have permitted the development of a new wave of tests and disciplines that can be considered within the concept of biomarkers. These include transcriptomic, proteomic and metabolomic tests which are being developed. Unfortunately, an announcement that a new technology is available does not mean that it has a good clinical use. In psychiatry, for example, limited data exist to support the use of blood cell expression to ably represent brain cell expression [28]. Current psychopharmacological knowledge suggests that peripheral markers, such as blood expression, may not be good biomarkers in pharmacogenetic studies for measuring brain neuron response to psychiatric treatments. Transporters at the blood-brain barrier may have an important role in the response to psychiatric drugs. Using peripheral biomarkers of psychiatric drug response may make more sense if one believes that some of the ADRs are related to peripheral mechanisms outside the brain, such as the possible direct influence of antipsychotics on lipid and glucose metabolism [29].

\section{The concepts of personalized medicine and personalized prescription in the literature}

The author has a preference for two terms usually related to pharmacogenomics and frequently used by lay journals: "personalized medicine" and "personalized prescription". "Personalized medicine" is described by Lesko [30] as "a comprehensive, prospective approach to preventing, diagnosing, treating, and monitoring disease in ways that achieve optimal individual health-care decisions." The idea of personalized medicine is that each individual may be different and needs to be treated differently by his/her physician. Ruaño [31] reminded us that physicians have traditionally practiced personalized 
medicine in their attempts to decide the best treatment for each of their patients. However, physicians were not using the term "personalized medicine" and the personalized approach traditionally used by physicians was probably based on subjective physician preferences and not on scientific knowledge. In the view of this author, personalized medicine is a very global concept that may include "personalized surgery", "personalized rehabilitation”, "personalized nutrition”, and other types of personalized medical interventions and, more importantly for pharmacologists, "personalized prescription."

"Personalized prescription" was defined by a Science editorial [32] as "tailoring drugs to a patient's genetic makeup". That editorial in 1997 on the research horizons for 1998 predicted that personalized prescription would "soon" reach clinical practice [32]. Other more precise estimates for the generalized use of personalized prescription have been advanced: 2015 by Time magazine [33] and 2020 by $J A M A$ [34]. If the generalized medical use of personalized prescription is going to occur in 7-12 years, one should notice preliminary steps toward its occurrence in the first generation of pharmacogenetic tests available in psychiatry (see Section 10). Even business journals [35] describe psychiatry (along with oncology) as being at the forefront in the use of pharmacogenomics in medicine. In the real world, the concept of personalized prescription has been wider than pharmacogenetics or pharmacogenomics. As a matter of fact, the first versions of two personalized prescription tests, for Trastuzumab (Herceptin) [36] and TPMT [37], usually did not include genotyping.

\section{A personal view of personalized prescription as a concept extending beyond pharmacogenetics}

In the opinion of the author [38], personalized medicine should include not only the use of biomarkers that may or may not be pharmacogenetic tests, but the consideration of all scientific information valid for prescribing medication. Pharmacology is a mechanistic science and knowing the pharmacological principles behind the response of a drug allows predictions to be made. For many drugs, genetic factors may be irrelevant in drug response or much less important than other non-genetic factors. Our pharmacological knowledge of each drug should determine what aspects are important in personalizing the prescription of that drug. In this comprehensive view of personalized prescription, 
clinicians need to consider genetic, environmental and personal variables when prescribing any medication [38]. If there are important genetic variables in the response to a specific drug they can be explored by pharmacogenetic tests. Environmental variables such as co-medication, herb supplements, foods, beverages, and smoking may be much more important than genetic factors for some drugs. Personal factors such as age, gender, or medical illnesses (renal or hepatic insufficiency) may be crucial personal variables in the response to some other drugs. Any classificatory attempt has to face the complexity of and the lack of boundary between medical concepts [39], as there are no perfect definitions of these genetic, environmental or personal factors affecting drug response. Genetic factors in drug response can be defined as those requiring a pharmacogenetic test for identification. Environmental factors come from outside the individual and are usually temporary. Personal factors are relatively stable characteristics in a specific individual and usually recorded in the patient's medical history.

\section{Personalized prescription should incorporate pharmacokinetic and pharmacodynamic}

\section{knowledge}

In the view of the author, to better apply the principles of personalized prescription one needs to understand the influence of genetic, environmental and personal factors on the pharmacokinetics and pharmacodynamics of each drug. Next is a short summary focused on antipsychotics, antidepressants, mood stabilizers and benzodiazepines.

\subsection{Pharmacokinetics of psychiatric drugs: a brief summary}

Our knowledge of the pharmacokinetics of psychiatric drugs is relatively well developed in the area of the Phase I metabolic enzymes (oxidation enzymes). Most psychiatric drugs are metabolized by the cytochrome P450 (CYP) enzymes. Genetics are particularly important for two polymorphic CYPs, the cytochrome P450 2D6 (CYP2D6) and the cytochrome P450 2C19 (CYP2C19). Unusual metabolizers, including both poor metabolizers (PMs), who do not have active enzyme, and ultrarapid metabolizers (UMs), who have too much active enzyme, have been described for both CYP2D6 and CYP2C19 [40,41]. Some rare subjects $(<1 / 1000)$ are PMs for both CYP2D6 and CYP2C19 [42]. Section 9 describes the first 
attempts to take CYP genotyping to the clinical environment. The cytochrome P450 1A2 (CYP1A2) and the cytochrome P450 3A (CYP3A) are also important for psychiatric drugs. They are heavily influenced by the environment and PMs and UMs for these CYPs do not exist or are very rare $[40,43]$.

The CYP2D6 is probably a peculiar gene, since environmental influences on its activity are very limited. Normal CYP2D6 metabolizers, called extensive metabolizers (EMs), may look like PMs if they are taking a powerful inhibitor (e.g., fluoxetine, paroxetine, or bupropion) because the CYP2D6 is completely inhibited. CYP2C19, CYP1A2 and CYP3A can be inhibited and induced. CYP2D6 metabolism is important for some antipsychotics and some antidepressants [41]. CYP2C19 metabolism is important for some antidepressants and some benzodiazepines [41]. CYP3A metabolism is important for some antipsychotics, some antidepressants, some benzodiazepines and some mood stabilizers [43].

Of the Phase II metabolic enzymes (conjugation enzymes), the Uridine 5'- diphosphate glucuronosyltransferases (UGTs) are the most important enzymes for psychiatric drugs, including some antipsychotics, some antidepressants, some mood stabilizers and some benzodiazepines [44]. UGTs are less well understood and are neglected when compared with CYPs. Factors that contributed to this neglect include: 1) the overlapping activity of UGTs and the lack of selective probes, 2) the complexity of the glucuronidation cycle, and 3) the difficulty of developing analytic methods to measure glucuronides.

Transporters may be important, too. The P-glycoprotein, P-gp, is one such transporter. P-gp is an ATP-dependent efflux pump that is involved in the blood-brain barrier and may be important for absorption since it is located in the small intestine. P-gp has substrates, inducers and inhibitors that overlap with CYP3A. Several, but not all, antidepressants [45], antipsychotics [46], and possibly anticonvulsants/mood stabilizers [47] are substrates of this transporter to different degrees, suggesting that there may be differences between blood and brain concentrations, at least with some of these compounds. Moreover, there may be differences between a compound and its metabolites and, in the case of risperidone and its metabolite [48], this may contribute to differences in their ADRs and dosing [49, 50]. Renal excretion is important for some psychiatric drugs, particularly lithium, and may have some 
influence on risperidone and paliperidone clearance. A new generation of transporters may be important in renal excretion, but there is limited knowledge of their relevance for psychiatric drugs $[51,52]$.

Ideally, for each drug the contribution of genetic, environmental and personal variables should be taken into account when trying to predict dosing, but the literature only offers the initial attempts of pharmacokinetic models to estimate the relevance of these variables for psychiatric medications [53-57].

\subsection{Pharmacodynamics of psychiatric drugs: a brief summary}

The honest truth is that we do not have a clear understanding of how psychiatric drugs work. When using the same drug for different indications, pharmacokinetic variables may be common across psychiatric disorders but we are not sure that is true for pharmacodynamic targets, too. SSRIs are always metabolized in the same way independently of patient diagnosis, but SSRI pharmacodynamics may or may not be the same in depression as in anxiety disorders.

It is currently believed that the efficacy of antipsychotics is mainly explained by the blockade of dopamine D2 receptors. Some ADRs such as hyperprolactinemia and reversible extrapyramidal side effects are also mainly explained by D2 blockade [49]. Other frequent ADRs may be explained by the blockade of other receptors. Orthostatic hypotension may be explained by the blockade of $\alpha$ adrenergic receptors. Sedation is probably explained by the blockade of histamine and/or muscarinic receptors. Weight gain is probably explained by the blockade of histamine and/or serotonin $2_{\mathrm{C}}$ receptors [49]. Constipation and dry mouth are probably explained by antimuscarinic properties. Other ADRs appear to have peripheral mechanisms, including disturbances in lipid and glucose metabolism and QTc increases in the heart [49]. Different antidepressants appear to work in different ways. Some are both serotonin and noradrenaline reuptake inhibitors; others are selective serotonin or noradrenaline reuptake inhibitors; others appear to influence multiple serotonin receptors or are monoamine oxidase inhibitors (MAOI) [58]. The brain receptors behind some antidepressant ADRs are similar to those of antipsychotics (adrenergic, muscarinic and histaminic receptors), but when combined with other serotonin drugs many antidepressants risk seriously disturbing the serotonin system by causing what is called serotonin 
syndrome. Benzodiazepines are thought to act mainly by binding to $\mathrm{GABA}_{\mathrm{A}}$ receptors and increasing their affinity to GABA (allosteric modulators) [59]. The mood stabilizer mechanism of action is poorly understood; they may act through the cell-signaling pathways, the second messenger system [60].

One has to acknowledge that when compared with pharmacokinetic studies, pharmacodynamic studies in psychiatry present an additional level of complexity. The in vivo activity of pharmacokinetic factors can be explored by measuring blood levels. The only question left is how well blood levels reflect brain concentrations at important sites. The in vivo activity of pharmacodynamic factors can only be explored indirectly by brain imaging studies. These studies are limited by the expense and complexity of the technology and are characterized by small sample sizes; therefore, the information on genetic, environmental and personal variables in pharmacodynamics is almost non-existent. The clinical literature suggests that drug interactions, aging and some illnesses (e.g., Parkinson's disease for antipsychotics) are important environmental influences on pharmacodynamic targets [49].

The literature on the influence of genetics on pharmacodynamic variables for psychiatric drugs has presented major problems with replication. Section 10 describes the first attempt to create a pharmacogenetic efficacy test. The possibility of an association between weight gain and serotonin receptor variations may be one of the more promising leads, although it is far from clinical practice [61]. Section 10 also mentions the first attempt to develop pharmacogenetic testing for antidepressant pharmacodynamics. The complexity of mood stabilizer response makes pharmacogenetic efficacy testing an unlikely development in the near future [60]. There has been little interest in clinical studies on the pharmacogenetics of benzodiazepine response.

The literature [41] is beginning to suggest that the genetics of pharmacodynamic targets may be much more complicated than the genetics of pharmacokinetic factors. Recently, Nebert et al [18] have stressed the relevance of gene characteristics by proposing that pharmacokinetic genes tend to be highpenetrance and predominantly monogenic, while pharmacodynamic genes tend to be more polygenic. Similarly, Maier and Zobel [62] stressed the importance of gene effect sizes by proposing that 
pharmacodynamic targets display a genetic influence of unknown magnitude emerging from the activity of multiple genes, each with only a small effect; on the other hand, pharmacokinetics reveals a documented strong genetic determination, which is mainly influenced by variants in a few genes.

\section{Personalized prescription should pay attention to therapeutic window and prescriber's role}

Drugs are approved in well-controlled studies that recommend an average dose and a range. These controlled studies increase the signal-to-noise ratio by reducing the noise; they exclude many patients and control treatment conditions. After drug approval physicians may utilize drugs differently than their use in clinical trials; therefore clinical practice brings more noise, including all kinds of patients, off-label use, co-medications, and varieties of doses and dosing schedules.

The last 15 years of drug development in psychiatry have been characterized by the transition from old, somewhat "toxic" drugs such as lithium, first-generation antipsychotics and tricyclic antidepressants (TCAs), to drugs that are much safer and have much wider therapeutic windows. In the opinion of the author, these pharmacoepidemiological facts are determinative in reference to the possibilities for success of personalized medicine in the clinical environment. The use of wider therapeutic window drugs makes the development of personalized medicine more difficult.

When physicians use toxic drugs with narrow therapeutic windows, they are severely restricted by the pharmacological makeup of the drugs, particularly their pharmacokinetics. High doses are toxic. Thus, drug pharmacology and pharmacokinetics are powerful signals for the older psychiatric drugs, limiting their noise. Using pharmacokinetic variables to personalize dosing of narrow therapeutic window psychiatric drugs has a high potential of being successful, due to the lower level of noise.

When doctors use newer drugs with wide therapeutic windows, they are freer to use wide dose ranges since they see little or no toxicity. In these situations doctor preferences or biases may be much more important than pharmacology in determining dose or drug selection [63]. When drug dosing is heavily influenced by physician choice it is much harder to predict the effects; thus the system has a lot of noise and the pharmacological signals may be lost in the noise [63]. Therefore, personalized dosing of 
wide therapeutic window drugs may be quite difficult to develop or be completely irrelevant, since dosing in the real world of clinical practice may have little relevance in predicting drug response. The most complex cases are the wide therapeutic window drugs that also inhibit their own metabolism, such as paroxetine, fluoxetine or fluvoxamine [64]. They are poor candidates for personalized dosing [38, 65].

\section{Safety personalized prescription should pay attention to the mechanism behind ADRs}

ADR types are important and have not received enough attention in the pharmacogenetic literature. There are two main types of ADRs [66]. Type A (or pharmacological) refers to the augmentation of the pharmacologic action and is dose-dependent. Pharmacokinetic genes may be important for narrow therapeutic window drugs in that PMs will have ADRs. Pharmacodynamic genes may be important, too, for predicting Type A ADRs. Type B (or idiosyncratic) ADRs are not predicted by pharmacologic action. Two main mechanisms are suggested: peculiar idiosyncratic metabolic pathways leading to reactive metabolites and/or immunological response [67]. Pharmacokinetic genetic variations may be important for some of these type B ADRs, but this is unproven.

Some idiosyncratic ADRs are associated with the HLA system. HLA-B ${ }^{*} 1502$ is strongly associated with the carbamazepine-induced Stevens-Johnson syndrome/toxic epidermal necrolysis in Han Chinese [68]. Genotyping for the HLA-B*1502 allele in persons with South Asian ancestry is FDArecommended [69].

\section{Personalized prescription can be implemented as personalized drug selection and/or as}

\section{personalized dosing}

The author believes that personalized prescription in the clinical environment can be expressed in two main ways: as personalized selection of the drug and as personalized dosing.

\subsection{Personalized drug selection}

Personalized selection of the drug has three levels: the first requires excluding some specific drugs in some unusual patients, the second requires matching some groups of drugs to some group of patients for purposes of greater efficacy or safety, and the third requires finding the best drug for each patient. 
The first level of personalized drug selection is not controversial (Table 1) and refers to what has traditionally been referred to in medical practice as drug contraindication. The majority of the drug package inserts developed in the last 10 years provided information regarding safety contraindications that frequently include environmental and personal variables. An example of using an environmental variable to exclude a drug would be a drug-induced increasing QTc, contraindicating ziprasidone [70], and indicating the use of another antipsychotic with less risk of increasing QTc. An example of personal contraindication is to exclude psychiatric drugs with teratogenic potential in pregnant women. Only recently has the FDA focused on utilizing genetic tests to exclude drugs; using HLA-B*1502 genotyping to exclude carbamazepine, as described in the prior section, is a clear example. The author believes it is likely that other similar pharmacogenetic testing (or other biomarkers) may be developed in the near future, preventing some rare idiosyncratic ADRs. The main limitation in preventing rare idiosyncratic ADRs is the development of large pharmacological databases by interested research groups, some international, which may allow the identification of a sufficient number of these rare subjects. These databases are being developed for particular diagnoses [71]. An alternative to research groups focused on a peculiar idiosyncratic ADR is to conduct even larger studies by developing large DNA banks from multiple recruiting sites, collecting clinical information on ADRs in a systematic and unified way [72,73].

The second level of personalized selection includes exclusion of some drugs in some patients due to frequent ADRs or lack of efficacy. An example of a personal variable used to personalize antipsychotic selection would be that of using older age in females to exclude antipsychotics with a high risk of producing tardive dyskinesia, since older women have a much higher risk (e.g., one of our studies showed that the risk is 3 times as high in older women; their odds ratio was 3.0) [74]. An example of an environmental variable used to personalize antipsychotic selection is the use of weight gain to dictate prescription of an antipsychotic with a low risk of weight gain for the patient taking other drugs that increase appetite (e.g., lithium or valproic acid). As pharmacology, both pharmacodynamics and pharmacokinetics, varies across drugs, this second level of personalized drug selection has a reasonable 
possibility of being successful. The main problem impeding its success is that the development of reliable tests for personalized drug selection is expensive. In the field of antipsychotics an interesting development has occurred in the area of cost-effectiveness. The second generation of antipsychotics is much more expensive than the old antipsychotics $[75,76]$. But now it is becoming clear that second-generation antipsychotics may not be better, and certainly they are less cost-effective [77, 78]. First-generation antipsychotics generate more risk of tardive dyskinesia (particularly those with high potency) and some may promote metabolic syndrome (particularly those with high potency). A reasonable alternative from the public health point of view may be to use the cheaper antipsychotics with pharmacogenetic tests that decrease their ADRs. The difference in cost (hundreds versus thousands of dollars per year) may accommodate a few hundred dollars for pharmacogenetic testing. This testing may be CYP-based or new tests may be developed that could be used in the prevention of tardive dyskinesia [79] or metabolic syndrome $[80,81]$. However, US society may not want to go back to older, cheaper drugs [76].

The third level of personalized selection, which includes choosing the best drug for the average patient, is much more controversial and difficult (Table 1). The controversy is that important economic benefits may be derived for most frequently selected drug. The difficulty is that selection of a previously unused drug is a very complicated process since it requires considering risk versus benefits, safety versus efficacy. The author has no non-controversial examples of personal or environmental variables that can help at this level of personalizing drug selection. In summary, the personalized selection of a drug for the majority of patients may be a very difficult issue to implement in the clinical environment, due to the economic implications and also to the complexity of developing methods balancing both safety and efficacy. As indicated in Section 6, although safety mechanisms may be common to various indications, efficacy mechanisms may vary for the same drug for different indications.

\subsection{Personalized dosing}

Once a specific drug is selected, using pharmacology for the implementation of personalized dosing is easier; pharmacodynamic and pharmacokinetic knowledge of dosing is clear. 
The experience of the author suggests that practicing clinicians can easily understand personalized dosing using pharmacokinetic principles as long as the drug follows linear kinetics. Many psychiatric drugs follow linear kinetics, and that probably includes antipsychotics [43]. As previously indicated, fluoxetine, paroxetine and fluvoxamine are auto-inhibitors; therefore they do not follow linear kinetics. Some of the mood stabilizers that are auto-inducers, such as carbamazepine or lamotrigine, may not follow linear kinetics and their kinetics change over time. The beauty of linear kinetics is that concentration (C) and dose (D) follow a linear relationship with a stable C/D ratio. Thus, if one increases D by 2, C as an average will increase by 2 and if one decreases D by 2, C as an average will decrease by 2. Wong et al. [82] reported that phenytoin increases the oral clearance of quetiapine by 5; this would require, on average, a fivefold increase of $\mathrm{D}$ to maintain the same $\mathrm{C}$. If one uses an average quetiapine $\mathrm{D}$ of $700 \mathrm{mg} /$ day, to compensate for phenytoin the average $\mathrm{D}$ would need to be at least $3500 \mathrm{mg} /$ day.

Pharmacodynamic factors may also be important in personalizing dosing. The dopamine D2 receptors (DRD2) are definitely important in explaining antipsychotic-induced EPS. Let us imagine that a genetic abnormality interferes with the DRD2 gene. This would certainly increase the risk of EPS. Unfortunately, no such genetic variation has been identified [61].

Table 1 describes two levels of complexity in personalizing dosing. These two levels are based on a drug's therapeutic window. The narrower the therapeutic window (or index) of the drug, the more important personalized dosing becomes.

A narrow therapeutic window means that once we have reached an efficacious $\mathrm{D}$, it is relatively easy to move to the unsafe D. In narrow therapeutic window drugs, pharmacodynamic variables may be important in personalizing dosing regarding safety but pharmacokinetic variables are definitely important in that regard. In narrow therapeutic window drugs, the influence of genetic, environmental and personal factors on pharmacokinetic factors may make the patient behave as a PM. This means that with unexpectedly low $\mathrm{D}$ he/she may have a high $\mathrm{C}$. The way to correct an increased $\mathrm{C} / \mathrm{D}$ ratio is by lowering D. High C determines only the increased risk of Type A ADRs; it does not determine the specific ADR. 
Although it has not been studied, the effects of genetic (or environmental or personal) variability on pharmacodynamics may determine the specific ADR. For example, the presence of a high C/D ratio in a patient taking R can be explained by CYP2D6 PM status (a genetic reason); use of paroxetine, a powerful CYP2D6 inhibitor (an environmental reason); or renal insufficiency (a personal reason), each of which decreases R elimination. All of them promote high R C. We cannot predict which ADRs may manifest. Any of the frequent R ADRs are possible: extrapyramidal symptoms (EPS), sedation, orthostatic hypotension, or gastrointestinal symptoms. The presence of one or another may be explained by pharmacodynamic factors including genetics.

If the drug has a very wide therapeutic window, personalized dosing may not be too relevant; dosing may have relatively little influence on safety and efficacy. In the real world, the prescriber's decision concerning dosage may be much more important than pharmacology, thus complicating the process of implementing personalized dosing using biomarkers or pharmacogenetic tests. As previously indicated, efficacy biomarkers may be difficult to develop. Safety biomarkers, including pharmacogenetic testing for personalized dosing, may have some potential for wide therapeutic window drugs if dosing influences the presence of ADRs in the clinical environment.

Due to limited space, this perspective article cannot explain how personalized dosing may be of practical use in the real world. Prior articles focused on how genetic, environmental and personal variables influence the pharmacokinetics and pharmacodynamics of risperidone $[49,50]$ and how pharmacogenetic testing and blood levels can be used to predict risperidone dosing [54].

\section{Pharmacogenetic testing in psychiatry at present}

The current status of pharmacogenetic testing in psychiatry, which was the focus of two prior articles $[83,84]$, does not look particularly promising. Of the five tests described in peer-reviewed publications, the one which focused on using antipsychotics safely in regard to metabolic syndrome is not on the market, but the company hopes to seek FDA approval in the future (PhyzioType test) [80]. Another of the five tests focused on idiosyncratic ADRs and was on the market for less than a year, due to 
insufficient sensitivity and specificity (PGxPredict:CLOZAPINE) [85]. The third was an efficacy test (LGC clozapine test) using only British subjects [86], meaning that replication in other subjects may be an issue [61]. Finally, lack of clinician use appears to be an issue for the two marketed CYP tests [87], one of which only measures CYP2D6 PMs and CY2C19 PMs well (Luminex Tag-It ${ }^{\mathrm{TM}}$ Mutation Detection Kit). The other (the AmpliChip CYP 450 Test) includes more alleles, particularly those associated with the CYP2D6 UM phenotype, and is more expensive [86]. However, the clinical use of these two CYP tests is limited in that the older drugs with narrow therapeutic windows (TCAs and typical antipsychotics) were not included, and they are of limited use for new drugs (perhaps for risperidone). The two CYP tests are also limited by the sparse clinical evidence available $[41,88]$ and by lack of psychiatrist education [89]. The use of serotonin transporter gene promoter variation to predict antidepressant response may be doomed by the complexity of the clinical issues and by lack of knowledge of the phenotype-genotype relationship [84]. An attempt to resuscitate an unmarketed psychiatric drug iloperidone, using pharmacogenomic testing, has not been successful [84].

\section{The future of pharmacogenetic testing in psychiatry}

Let's start with the positive aspects as we look toward the future, namely, that genetic technology continues to surprise with meaningful new advances that may have relevance for testing in the clinical environment. A new nanotechnology can provide genotyping results in a few hours, providing busy clinicians with rapid answers [90]. The FDA approved a warfarin test using this technology in September 2007 [91]. It is currently unclear how epigenetic changes can be tested in the clinical environment, but Marsh [92] suggested that pyrosequencing may be a flexible technology that can be used for testing SNPs, CNVs, and also methylation status.

HLA-B*1502 genotyping in Asians, a test developed in neurological patients, should be used in psychiatric patients taking carbamazepine. The role of this test is very limited; it is a pharmacogenetic test for one drug and for one racial group, and only eliminates the risk of relatively rare idiosyncratic ADRs. Clinicians have complained to this author that such an advance is miniscule. Unfortunately this is the only 
pharmacogenetic test in the immediate future of psychiatry that has definitive support for its clinical indication. It is possible that some other test for the detection of some other unusual idiosyncratic reaction to psychiatric drugs in a racial group may be developed in the next five years.

Currently, CYP2D6 and CYP2C19 genotyping appear to have little future (see the next section for possible reasons). Pharmaceutical companies are eliminating drugs metabolized by CYP2D6 from their pipeline. As indicated in Section 9, first-generation antipsychotics may be as efficacious as the new ones. As they are much cheaper, marketing the use of first-generation antipsychotics plus personalized tests may be the way to go. Unfortunately, this idea is contrary to the current marketing strategies of the pharmaceutical companies which are promoting second-generation antipsychotics.

This article defines a new way of looking at personalized prescription, describing it as the use of genetic, environmental or personal information for selecting drugs and/or prescribing dosages. It also proposes that, in the case of many drugs, personalized prescription is likely not to include genetic testing. With this broad definition, personalized prescription can be utilized. It requires only that sophisticated clinicians understand that genetic, environmental or personal variables influence pharmacokinetic and pharmacodynamic response; the therapeutic window of the drug may be important, too. Blood levels, currently called therapeutic drug monitoring (TDM), have been used by psychiatrists to personalize dosing for lithium, TCAs and some antipsychotics [93]. Unfortunately, all of these are old drugs rarely used by young prescribers in psychiatry. The marketing of the new drugs has convinced psychiatrists that they do not need to use these old drugs, thereby using TDM in psychiatry appears irrelevant. It also makes teaching this broad view of personalized prescription difficult.

\section{The limited future of pharmacogenetic testing in psychiatry}

Every year for the past five years, this author has become more pessimistic regarding personalized prescription. He further ruminates that there is not much "future" for it in the next five years in psychiatry; only small incremental steps appear possible. The obstacles to progress for personalized prescription in psychiatry appear insurmountable. These obstacles have been reviewed in prior articles 
$[82,83,86,88]$ and include: 1$)$ the lack of interest among funding agencies for pharmacogenetic studies in the real world; 2) the lack of enthusiasm among pharmaceutical companies for pharmacogenetic testing; 3) the difficulty of publishing pharmacogenetic studies focused on real world questions; 4) financial issues (a new diagnostic test may have limited benefit, whereas a drug might become a blockbuster and make millions of dollars); 5) the lack of training for physicians in the use of the new pharmacogenetic tests; and 6) the lack of prior approval of pharmacogenetic tests, which means facing a different type of regulatory oversight than used for drug approval. This perspective article emphasizes 7) the complexity of the methodological/scientific issues in personalized prescription; added to the prior six obstacles. This complexity has led to the virtual absence of controlled studies in the real world designed to explore pharmacogenetic testing in psychiatric clinical practice.

Pharmacogenetics appears to be progressing faster in psychiatry than in other areas of medicine, with the possible exception of oncology. However, when psychiatry is compared with oncology, oncology certainly appears to have a brighter future in terms of using biomarkers to personalize prescription because oncology has easy access to tissue, as well as a better understanding of the complex pathophysiological mechanism. Not all of these biomarker tests for personalized prescription will be pharmacogenetic tests. As a matter of fact, some of the tests used in the clinical practice of oncology for personalizing prescriptions are not pharmacogenetic tests. Cancer's lack of stigma (compared with the stigma associated with mental illness) and the lethality of the oncological diseases, combined with the high toxicity and the high cost of oncological drugs, may make personalized prescription in oncology easier to sell and more cost-effective than in psychiatry. As a matter of fact, CYP2D6 genotyping may be getting a second life in oncology since tamoxifen may not have protective effects against breast cancer in CYP2D6 PMs [94].

This perspective article further elaborates on a previously neglected obstacle, the complexity of the scientific task of resolving the conceptual issues behind personalized prescription. Currently, the author thinks that, in psychiatry, pharmacogenetic tests or other types of complex biomarkers have some 
potential in two areas: 1) excluding the use of some drugs for some unusual patients (has major potential since neurology provided the first pharmacogenetic test for carbamazepine), and 2) personalizing drug dosing by using pharmacokinetic genes in narrow therapeutic window drugs (has some potential but these drugs may be irrelevant for clinical practice unless the old antipsychotics are returned to use). There is dubious potential for: 1) selecting some drugs within a class due to ADR or efficacy profile, and 2) selecting dosing in a wide therapeutic window drug. The author thinks that there is no short-term potential in finding the best drug for each patient. This "very sophisticated" level of personalized prescription is beyond our current knowledge and study methodologies.

\section{Conclusions}

In conclusion, this perspective article has an "ambivalent" message. The pessimistic aspect is that the future of pharmacogenetic testing or other biomarkers in personalizing prescription in psychiatry does not look promising, other than the limited examples noted, which are not widely used. However, if one looks closely at both components of personalized prescription, personalized drug selection and personalized dosing, it is obvious that personalized dosing has immediate application if one understands it as the use of our current scientific knowledge of genetic, environmental and personal variables to determine dosing. Nevertheless, there is need to complicate the picture by bringing to bear other pharmacological concepts such efficacy versus safety, pharmacokinetics versus pharmacodynamics, narrow versus wide therapeutic window drugs, and dose-related versus idiosyncratic ADRs.

The optimistic aspect is that pharmacological information on drug-drug interactions and personal variables plus pharmacogenetic tests and TDM can currently be used to personalize dosing, particularly for narrow therapeutic window drugs. A major limiting factor is the lack of well-trained psychiatrists who use pharmacological principles to personalize prescription in psychiatry. These sophisticated psychiatrists will be needed in the future to incorporate new pharmacogenomic testing or other biomarker tests to personalize prescriptions in psychiatry. 


\section{References}

[1] McKusick VA. The anatomy of the human genome: A neo-vesalian basis for medicine in the $21^{\text {st }}$ century. JAMA 2001;286:2289-95.

[2] Collins FS, Green ED, Guttmacher AE, Guyer MS, on behalf of the US National Human Genome Research Institute. A vision for the future of genomics research. Nature 2003;422:835-47.

[3] Emery J, Hayflick S. The challenge of integrating genetic medicine into primary care. BMJ 2001;322:1027-30.

[4] Phillips KA, Veenstra DL, Oren E. Lee JK, Sadee W. Potential role of pharmacogenomics in reducing adverse drug reactions: A systematic review. JAMA 2001;286:2270-9.

[5] Fodor SP. Massively parallel genomics. Science 1997;277:393-5.

[6] Koch WH. Technology platforms for pharmacogenomic diagnostic assays. Nat Rev Drug Discov 2004;3:749-61.

[7] Steemers FJ, Gunderson KL. Whole genome genotyping technologies on the BeadArray platform. Biotechnol J 2007;2:41-9.

[8] Golden F, Lemonick MD. The race is over. Time 2000;July3:18-23.

[9] Lander ES, Linton LM, Birren B, Nusbaum C, Zody MC, Baldwin J, et al. Initial sequencing and analysis of the human genome. Nature 2001;409:860-921.

[10] Venter JC, Adams MD, Myers EW, Li PW, Mural RJ, Sutton GG, et al. The sequence of the human genome. Science 2001;291:1304-51.

[11] Subramanian G, Adams MD, Venter JC, Broder S. Implications of the human genome for understanding human biology and medicine. JAMA 2001;286:2296-307.

[12] Reichardt JKV. Quo vadis genoma? A call for pipettes for biochemists. Trends Biochem Sci $2007 ; 32 ; 529-30$.

[13] Reichardt JKV. Realizing the full potential of the sequenced human genome (letter). Trends Genet $2008 ; 24 ; 219-20$. 
[14] Redon R, Ishikawa S, Fitch KR, Feuk L, Perry GH, Andrews TD, et al. Global variation in copy number in the human genome. Nature 2006;444:444-54.

[15] Ouahchi K, Lindeman N, Lee C. Copy number variants and pharmacogenomics. Pharmacogenomics 2006;7:25-9.

[16] Ingelman-Sundberg M, Sim SC, Gomez A, Rodriguez-Antona C. Influence of cytochrome P450 polymorphisms on drug therapies: Pharmacogenetic, pharmacoepigenetic and clinical aspects. Pharmacol Ther 2007;116:496-526.

[17] Court MH. A pharmacogenomics primer. J Clin Pharmacol 2007;47:1087-103.

[18] Nebert DW, Zhang G, Vesell ES. From human genetics and genomics to pharmacogenetics and pharmacogenomics: Past lessons, future directions. Drug Metab Rev 2008;40:187-224.

[19] Wang Y, Krishnan HR, Ghezzi A, Yin JCP, Atkinson NS. Drug-induced epigenetic changes produce drug tolerance. PLoS Biol 2007;5:e265.

[20] Vogel F. Moderne probleme der Humangenetik. Ergeb Inn Med Kinderheild 1959;12:52-125.

[21] Pirmohamed M. Pharmacogenetics and pharmacogenomics. J Clin Pharmacol 2001;52:345-7.

[22] Roses AD. Pharmacogenetics and drug development: The path to safer and more effective drugs. Nat Rev 2004;5:645-56.

[23] Wagner JA. Strategic approach to fit-for-purpose biomarkers in drug development. Annu Rev Pharmacol Toxicol 2008;48:631-51.

[24] Kirkwood SC, Hockett RD Jr. Pharmacogenomic biomarkers. Dis Markers 2002;18:63-71.

[25] Lesko LJ, Atkinson AJ Jr. Use of biomarkers and surrogate endpoints in drug development and regulatory decision making: Criteria, validation, strategies. Annu Rev Pharmacol Toxicol $2001 ; 41: 347-66$.

[26] FDA. Guidance for Industry: Pharmacogenomic data submission. FDA 2005. http://www.fda.gov/cder/guidance/6400fnl.pdf (Viewed in April 2008). 
[27] Salerno RA, Lesko LJ. Pharmacogenomics data: FDA voluntary and required submission guidance. Pharmacogenomics 2005;5:503-5.

[28] Glatt SJ, Everall IP, Kremen WS, Corbeil J, Sásik R, Khanlou N, et al. Comparative gene expression analysis of blood and brain provides concurrent validation of SELENBP1 up-regulation in schizophrenia. Proc Natl Acad Sci U S A 2005;102:15533-8.

[29] de Leon J, Diaz FJ. Planning for the optimal design of studies to personalize antipsychotic prescriptions in the post-CATIE era: The clinical and pharmacoepidemiological data suggest that pursuing the pharmacogenetics of metabolic syndrome complications (hypertension, diabetes mellitus and hyperlipidemia) may be a reasonable strategy. Schizophr Res 2007;96:185-97.

[30] Lesko LJ. Personalized medicine: Elusive dream or imminent reality? Clin Pharmacol Ther 2007;81:807-16.

[31] Ruaño G. Quo vadis personalized medicine. Personalized Med 2004;1:1-7.

[32] Science. New research horizons. Science 1997;278:2039.

[33] Lertola J. Deciphering the code and what might come from it. Time 1999;Nov 8:68-9.

[34] Collins FS, McKusick, VA. Implications of the human genome project for medical science. JAMA 2001;285:540-4

[35] Cappell K, Arndt M, Carey J. Drugs get smart. Business Week 2005;September 5:76-85

[36] Emens LA. Trastuzumab: Targeted therapy for the management of HER-2/neu-overexpressing metastatic breast cancer. Am J Ther 2005;12:243-53.

[37] Corominas H, Baiget M. Clinical utility of thiopurine S-methyltransferase genotyping. Am J Pharmacogenomics 2004;4:1-8.

[38] de Leon J. The crucial role of the therapeutic window in understanding the clinical relevance of the poor versus the ultrarapid metabolizer phenotypes in subjects taking drugs metabolized by CYP2D6 and CYP2C19. J Clin Psychopharmacol 2007 ;27:241-5.

[39] Sadegh-Zadeh K. The prototype resemblance theory of disease. J Med Phil 2008;33:106-39. 
[40] Ingelman-Sundberg M, Sim SC, Gomez A, Rodriguez-Antona C. Influence of cytochrome P450 polymorphisms on drug therapies: pharmacogenetic, pharmacoepigenetic and clinical aspects. Pharmacol Ther 2007;116:496-526.

[41] de Leon J, Armstrong SC, Cozza KL. Clinical guidelines for psychiatrists for the use of pharmacogenetic testing for CYP450 2D6 and CYP450 2C19. Psychosomatics 2006;47:75-85.

[42] Johnson M, Markham-Abedi C, Susce MT, Murray-Carmichael E, McCollum S, de Leon J. A poor metabolizer for both Cytochrome P450 2D6 and 2C19 (CYP2D6 and CYP2C19): A case report on antidepressant treatment. CNS Spectrums 2006;11:757-60.

[43] de Leon J, Armstrong SC, Cozza KL. The dosing of atypical antipsychotics. Psychosomatics 2005;46: 262-73.

[44] de Leon J. Glucuronidation enzymes, genes and psychiatry. Int J Neuropsychopharmacol 2003;6, $57-72$.

[45] El Ela AA, Hartter S, Schmitt U, Hiemke C, Spahn-Langguth H, Langguth P. Identification of pglycoprotein substrates and inhibitors among psychoactive compounds - implications for pharmacokinetics of selected substrates. J Pharmacy Pharmacol 2004;56:967-75.

[46] Boulton DW, DeVane CL, Liston HL, Markowitz JS. In vitro P-glycoprotein affinity for atypical and conventional antipsychotics. Life Sci 2002;71:163-9.

[47] Bauer B, Hartz AMS, Pekcec A, Toellner K, Miller DS, Potschka H. Seizure-induced upregulation of P-glycoprotein at the blood-brain barrier through glutamate and cyclooxygenase-2 signaling. Mol Pharmacol 2008;73:1444-53.

[48] Wang JS, Ruan Y, Taylor RM, Donovan JL, et al: The brain entry of risperidone and 9hydroxyrisperidone is greatly limited by p-glycoprotein. Int J Neuropsychopharmacology 2004; $7: 415-9$

[49] de Leon J, Armstrong SC, Cozza KL. A preliminary attempt to personalize risperidone dosing using drug-drug interactions and genetics. Part I. Psychosomatics 2008;49: 258-70. 
[50] de Leon J, Armstrong SC, Cozza KL. A preliminary attempt to personalize risperidone dosing using drug-drug interactions and genetics. Part II. Psychosomatics 2008;49:347-361 .

[51] Dubuske LM. The role of p-glycoprotein and organic anion-transporting polypeptides in drug interactions. Drug Safety 2005;28:789-801.

[52] Endres CJ, Hsiao P, Chung FS, Unadkat JD. The role of transporters in drug interactions. Eur J Pharm Sci 2006;27:501-17.

[53] de Leon J, Susce MT, Pan RM, Fairchild M, Koch W, Wedlund PJ. The CYP2D6 poor metabolizer phenotype may be associated with risperidone adverse drug reactions and discontinuation. J Clin Psychiatry 2005;66:15-27.

[54] de Leon J, Susce MT, Pan RM, Wedlund PJ, Orrego ML, Diaz FJ. A study of genetic (CYP2D6 and $\mathrm{ABCB} 1$ ) and environmental (drug inhibitors and inducers) variables that may influence plasma risperidone levels. Pharmacopsychiatry 2007;40:93-102.

[55] Diaz FJ, Santero V, Spina E, Cogollo M, Rivera TE, Botts S, et al. Estimating the size of the effects of co-medications on plasma clozapine concentrations using a model that controls for clozapine doses and confounding variables. Pharmacopsychiatry 2008;41:81-91.

[56] Botts S, Diaz FJ, Santoro V, Spina E, Muscatello MR, Cogollo M, et al. Estimating the effects of co-medications on plasma olanzapine concentrations by using a mixed model. Prog NeuroPsychopharmacology Biol Psychiatry 2008;32:1453-8.

[57] Reimers A, Skogvoll E, Sund JK, Spigset O. Drug interactions between lamotrigine and psychoactive drugs: Evidence from a therapeutic drug monitoring service. J Clin Psychopharmacol $2005 ; 25: 342-8$.

[58] Preskorn S. Outpatient management of depression: a guide for the primary-care practitioner. Professional Communications, Inc, Caddo, OK, 1999. 
[59] Reynolds DS. The value of genetic and pharmacological approaches to understanding the complexities of GABA(A) receptor subtype functions: The anxiolytic effects of benzodiazepines. Pharmacol Biochem Behav 2008;90:37-42.

[60] de Leon J. Pharmacogenetics of mood stabilizers in bipolar disorder. Eur Neuropychopharmacol 2008;18(Suppl 4):S197.

[61] Arranz MJ, de Leon J. Pharmacogenetics and pharmacogenomics of schizophrenia: A review of the last decade of research. Mol Psychiatry 2007;12:707-47.

[62] Maier W, Zobel A. Contribution of allelic variations to the phenotype of response to antidepressants and antipsychotics. Eur Arch Psychiatry Clin Neurosci 2008;258(Suppl 1):12-20.

[63] Botts S, Littrell R, de Leon J. Variables associated with high olanzapine dosing in a state hospital. J Clin Psychiatry 2004;65:1138-43.

[64] Hiemke C, Härtter S. Pharmacokinetics of selective serotonin reuptake inhibitors. Pharmacol Ther 2000;85:11-28.

[65] de Leon J. The potential of genotyping (letter). Science 2008;321:769.

[66] Pirmohamed M, Breckenridge AM, Kitteringham NR, Park BK. Adverse drug reactions. BMJ 1998;316:1295-8.

[67] Uetrecht J. Idiosyncratic drug reactions: Current understanding. Annu Rev Pharmacol Toxicol 2007;47:513-39.

[68] Chung WH, Hung SI, Hong HS, Hsih MS, Yang LC, Ho HC, et al. Medical genetics: A marker for Stevens-Johnson syndrome. Nature 2004;428:486.

[69] FDA. Information for Healthcare Professionals: Carbamazepine (marketed as Carbatrol, Equetro, Tegretol, and generics). FDA 2007.

\section{http://www.fda.gov/cder/drug/InfoSheets/HCP/carbamazepineHCP.htm}

[70] Pfizer. Geodon ${ }^{\mathrm{R}}$ (ziprasidone $\mathrm{HCl}$ ), Geodon ${ }^{\mathrm{R}}$ (ziprasidone mesylate) for injection for IM use only prescribing information. New York: Roering Division of Pfizer, Inc., March 2007. 
[71] Mockenhaupt M, Viboud C, Dunant A, Naldi L, Halevy S, Bavinck JNB, et al. Stevens-Johnson syndrome and toxic epidermal necrolysis: Assessment of medication risks with emphasis on recently marketed drugs. The EuroSCAR-Study. J Invest Dermatol 2008;128:35-44.

[72] Gurwitz D, Lunshof JE, Altmann RB. A call for the creation of personalized medicine databases. Nature Rev 2006;5:23-6.

[73] Ozdemir V, Williams-Jones B, Cooper DM, Someya T, Godard B. Mapping translational research in personalized therapeutics: From molecular markers to health policy. Pharmacogenomics 2007;8:177-85.

[74] de Leon J, Susce MT, Pan RM, Koch W, Wedlund PJ. Polymorphic variations in GSTM1, GSTT1, PgP, CYP2D6 CYP3A5, and dopamine D2 and D3 receptors and their association with tardive dyskinesia in severe mental illness. J Clin Psychopharmacol 2005;25:448-56.

[75] Duggan, M. Do new prescription drugs pay for themselves? The case of second-generation antipsychotics. J Health Econ 2005;24:1-31.

[76] Rosenheck RA, Leslie DL, Doshi JA. Second-generation antipsychotics: Cost-effectiveness, policy options, and political decision making. Psychiatr Serv 2008;59:515-20.

[77] Stroup TS, Lieberman JA, McEvoy JP, Swartz MS, Davis SM, Capuano GA, et al. Effectiveness of olanzapine, quetiapine, and risperidone in patients with chronic schizophrenia after discontinuing perphenazine: a CATIE study. Am J Psychiatry 2007;164:415-27.

[78] Sikich L, Frazier JA, McClellan J, Findling RL, Vitiello B, Ritz L, et al. Double-blind comparison of first- and second-generation antipsychotics in early-onset schizophrenia and schizo-affective disorder: findings from the Treatment of Early-Onset Schizophrenia Spectrum Disorders (TEOSS) study. Am J Psychiatry. 2008 Sep 15. [Epub ahead of print]

[79] Ozdemir V, Aklillu E, Mee S, Bertilsson L, Albers LJ, Graham JE, Caligiuri M, et al. Pharmacogenetics for off-patent antipsychotics: reframing the risk for tardive dyskinesia and access to essential medicines. Expert Opin Pharmacother 2006;7:119-33. 
[80] Ruaño G, Goethe JW, Caley C, Woolley S, Holford TR, Kocherla M, et al. Physiogenomic comparison of weight profiles of olanzapine- and risperidone-treated patients. Mol Psychiatry $2007 ; 12: 474-82$.

[81] de Leon J, Diaz FJ. Planning for the optimal design of studies to personalize antipsychotic prescriptions in the post-CATIE era: The clinical and pharmacoepidemiological data suggest that pursuing the pharmacogenetics of metabolic syndrome complications (hypertension, diabetes mellitus and hyperlipidemia) may be a reasonable strategy. Schizophr Res 2007;96:185-97.

[82] Wong YW, Yeh C, Thyrum PT. The effects of concomitant phenytoin administration on the steady-state pharmacokinetics of quetiapine. J Clin Psychopharmacol 2001;21:89-93.

[83] de Leon J, Arranz MJ, Ruaño G. Products for pharmacogenetic testing in psychiatry: A review of features and clinical realities. Clin Lab Med 2008; in press.

[84] de Leon J. Pharmacogenomics: the promise of personalized medicine for CNS disorders. Neuropsychopharmacol 2008 Sep 17. [Epub ahead of print].

[85] Malhotra A.K, Athanasiou M, Reed CR, Dain B, Carr J, Whalen H, et al. Discovery of genetic markers associated with clozapine induced agranulocytosis. Am J Med Gen Part B Neuropsychatr Gen 2005;138b:22.

[86] LGC. Clozapine response test: Pharmacogenetics in psychiatry. http://newsite.lgc.co.uk/our_science/pharmacogenetics.aspx (Viewed in October 2008).

[87] de Leon J. The AmpliChip CYP450 Test: Personalized medicine has arrived in psychiatry. Expert Rev Mol Diagn 2006;6:277-86.

[88] Kirchheiner J, Nickchen K, Bauer M, Wong M-L, Licinio J, Roots I, et al. Pharmacogenetics of antidepressants and antipsychotics: The contribution of allelic variations to the phenotype of drug response. Mol Psychiatry 2004;9:442-73.

[89] de Leon J, Susce MT, Murray-Carmichael E. The AmpliChip CYP450 Genotyping Test: Integrating a new clinical tool. Mol Diagn Ther 2006;10:135-51. 
[90] Lee HK, Lewis LD, Tsongalis GJ, Schur BC, Jannetto PJ, Wong SH, et al. Validation of a CYP2D6 genotyping panel on the NanoChip Molecular BiologyWorkstation. Clin Chem 2007;53:823-8.

[91] Nanosphere, Inc. Nanosphere announces FDA clearance of second molecular diagnostics assay. 2007, October 16. http://ir.nanosphere.us/phoenix.zhtml?c=214748\&p=irol$\underline{\text { newsArticle } \& I D=1075929 \& \text { highlight }}=($ Viewed in October 2008).

[92] Marsh S. Pyrosequencing applications. Methods Mol Biol 2007;373:15-24.

[93] Baumann P, Hiemke C, Ulrich S, et al: The AGNP-TDM expert group consensus guidelines: therapeutic drug monitoring in psychiatry. Pharmacopsychiatry 2004;37:243-56

[94] Beverage JN, Sissung TM, Sion AM, Danesi R, Figg WD. CYP2D6 polymorphisms and the impact on tamoxifen therapy. J Pharmaceutical Sci 2007;96:2224-31. 
Table 1. Levels of complexity of pharmacogenetic testing as an aid to personalized prescription in psychiatry

\section{PERSONALIZED DRUG SELECTION \\ 1.1. Exclusion of some drugs for some unusual subjects}

Easily done and non-controversial

Pharmacogenetic testing may identify individuals with high risk of idiosyncratic ADRs

Pharmacokinetic genetic testing may identify PMs taking pro-drugs (lack of efficacy)

Pharmacokinetic genetic testing may identify UMs taking pro-drugs (safety)

Pharmacodynamic genetic testing may identify variations associated with no response (lack of efficacy)

1.2. Exclusion of some drugs within one class in some patients due to frequent ADRs or lack of efficacy

To be developed: who will pay for the complex studies (better pharmacological understanding is needed)

Will cost differences between old drugs and new drugs pay for testing if used in clinical practice?

Unclear that pharmacokinetic genes have major effects (small effects are likely)

It is possible that pharmacodynamic genes may play a role but they need to be identified

\subsection{Selection of best drug for each patient}

Controversial: important economic benefits may be derived for most frequently selected drug

Public health point of view: Drug cost may need to be considered

Patient's point of view: Drug with good risk-benefit balance (safety-efficacy)

Unusual pharmacokinetic gene variations can be handled by dosing changes (see below)

Pharmacodynamic gene variations require balancing risk-benefit and safety-efficacy

It may not be easy to match individual genetic profiles and many different drugs

Models combining genetic, environmental and personal variables may be too complicated

It is not understood how prescribers' and patients' attitudes and beliefs influence drug selection Study replications may be a major problem (too much noise)

\section{PERSONALIZED DRUG DOSING}

\subsection{Drugs with narrow therapeutic window}

Easily done: recent package inserts include some "personalizing" information regarding safety

Using pharmacogenetic tests has potential but it is in its infancy:

i) PMs (genetic or taking inhibitors) need low doses to avoid ADRs (safety)

ii) UMs (genetic or taking inducers) need higher doses (efficacy)

Pharmacokinetic models need to consider genetic, environmental and personal variables

Pharmacodynamic genetic tests may be helpful for safety (decrease dosing): not clinically available

It is not known if pharmacodynamic genetic tests may be helpful for efficacy

\subsection{Wide therapeutic window drugs}

Not easily achieved: drug pharmacology may have small effects in determining dosing

Prescribers' attitudes and beliefs may introduce much noise in the system

PMs (genetic or taking inhibitors): may have limited relevance for avoiding ADRs (drugs are safe)

UMs (genetic or taking inducers): may only have relevance in extreme case (lack of efficacy)

Unknown whether pharmacodynamic genetics will be robust enough in tests for safety or efficacy

ADRs: adverse drug reactions. PMs: poor metabolizers. UMs: ultrarapid metabolizers. 